\title{
Role of biomaterials in neurorestoration after spinal cord injuries
}

\author{
Ioana Stanescu ${ }^{1 / 3}$, Gabriela Dogaru ${ }^{2 / 3}$
}

1. University of Medicine and Pharmacy "Iuliu Hatieganu" Cluj - Neuroscience Department 2. University of Medicine and Pharmacy "Iuliu Hatieganu" - Medical Rehabilitation and Physical Medicine Department

3. Rehabilitation Hospital Cluj

\begin{abstract}
Despite advances in knowledge and technology SCI remains one of the most severe and disabling disorders affecting young people. Spinal cord lesions result in permanent loss of motor, sensory and autonomic functions, causing an enormous impact on patient's personal, social, familial and professional life. There is currently no effective treatment available to improve severe neurologic deficits and to decrease disability.

Tissue-engineering techniques have developed a variety of scaffolds, made by biomaterials, used alone, incapsulated with cells or embedded with molecules, which are delivered to lesion site to achieve neural regeneration.

Biomaterials may provide structural support and/or serve as a delivery vehicle for factors to arrest growth inhibition and promote axonal growth. Biomaterials acts like cell-carriers for the injury site, but also as reservoirs for growth factors or biomolecules. Hydrogels are a promising therapeutical strategy in spinal cord repair. Nano-fibers provide a three-dimensional network, which mimic closely the native extracellular matrix, thus offering a better support for cell attachment and proliferation than traditional micro-structure.

New strategies like pharmacologic treatments, cell therapies, gene therapies and biomaterial tissue engineering should combine to increase their synergistic effect and to obtain the expected functional recovery in spinal cord injured patients.
\end{abstract}

Key words: spinal cord injury, biomaterials, neuroregeneration, combinatorial approach 


\section{INTRODUCTION}

Spinal cord injury (SCI) is caused mainly by traumatic factors, the leading cause being motor vehicle accidents, falls and violent attacks. According to the National Spinal Cord Injury Statistical Center (NSCISC) 2015, the incidence of SCI is estimated at 40 cases per million population in the United States, after exclusion of people who die at the scene of the accident. The average age at injury in now 42 years, $80 \%$ are males (1).

Most frequent neurologic deficit caused by SCI (after 2010) is incomplete tetraplegia - in $45 \%$ of cases, incomplete paraplegia in $21 \%$ of cases, complete paraplegia - $20 \%$ and complete tetraplegia in $14 \%$ of patients (1). Less than $1 \%$ of patients experienced complete neurological recovery after hospital discharge. The life expectancy for persons with SCI have not improved since the $1980 \mathrm{~s}$ and remain significantly below that of persons without SCI. Mortality rates are significantly higher during the first year after injury than during subsequent years, particularly for persons with the most severe neurological impairments (NSCISC, 2015) (1).

The severity of an injury is assessed by the five-level American Spinal Injury Association (ASIA), lesion, being included in A-E types and characterized as being complete or incomplete. Incomplete injuries have a better prognosis: $84 \%$ of patients with ASIA-C will improve to ASIA-D level and $66 \%$ of patients with ASIA-B level will be transformed into ASIA-C or D levels. Patients with motor complete lesions remain with irreversible deficits: only $7 \%$ of patients with ASIA-A level will improve to ASIA-B.

For these patients, improving neurologic deficits remains one the most important challenge for neuroscience research. Modern medical and surgical management have brought certain benefits in the outcome of SCI patients in acute and subacute phase, but in the chronic phase there is currently no effective treatment available to improve severe neurologic deficits (2).

The difficulty in achieving an efficient treatment plan for SCI patients is explained by the different types of mechanical injuries and by the complexity of the pathogenetic mechanisms involved.
Mechanical injuries could act as compressions, contusions or lacerations (2).

The pathogenesis of spinal cord injury is complex and aggressive, and is divided into primary and secondary injury mechanisms. Primary mechanisms are related to the mechanical impact and to its direct consequences on tissues: direct laceration, blood vessels disruption, hemorrhage, edema, necrosis, which trigger a cascade of local and general biochemical events: vasospasm, ischemia, membrane disfunctions, ionic disturbancies, neurotransmitter alterations. These events occur in acute phase (seconds to minutes after the injury) and continue in the subacute phase (minutes to weeks after the injury). The secondary injury mechanisms appear in the subacute phase and continue in the chronic phase (months to years after the injury). The first seconday event is inflammation, followed by further edema, excitotoxicity, free radical production, lipid peroxidation, neuronal apoptosis, demyelination, neurotransmitter disturbancies. These events lead to "cavitation": a fluid-filled cyst formed at the injury site after few days to weeks post-injury. The cyst is surrounded by reactive astrocytes, which form a glial scar and secrete inhibitory proteins for axonal re-growth (chondroitin sulfate proteoglycans). Even that the glial scar's role is to stabilize the damaged spinal cord, its effects are detrimental to neuroregeneration: the scar sets a physical and a chemical barrier to axonal regrowth and remyelination (2), (3).

The chronic phase is characterized by continued scar formation, Wallerian degeneration of injured axons and the development of cysts and/or syrinxes (4). It can be considered that at $\sim 1-2$ years postinjury, the neurological deficits have stabilized and the lesion has fully matured. The lesion itself is characterized by cystic cavitation and myelomalacia, representing the final stage of necrotic death after SCI. The good news for treatment in this phase came from the observation of Hulsebosch (5) that they are preserved axons in the subpial regions of the injured spinal cord, which could sustain recovery of neurologic functions. The bad news is that the spontaneous ability of the central nervous system (CNS) to regenerate is limited, 
due to an inhibitory environment generated at lesion site and due to the mechanical barrier of the glial scar. In chronic spinal cord lesions the obstacles for effective regeneration are the cystic cavity, the presence of inhibitory environment and the lack of growth factors. A complex therapeutic approach should bridge the cystic cavity, offering a structural support for cell and axon growth (as the extracellular matrix), should neutralize the inhibitory biomolecules, should add new cells to replace the lost ones and should secrete neurotrophic factors (2).

At this chronic stage, therapeutic strategies are aimed to encourage regeneration/sprouting of disrupted axons, promote plasticity with rehabilitation strategies, and improve the function of demyelinated axons with pharmacological measures or cellular transplantation substrates that may potentially remyelinate (4).

In recent years, tissue engineering using biomaterials and cell-based therapies using stem cell-transplantations are emerging strategies for neurorestoration after spinal cord injury. Various neurochemical and cellular repair strategies have been evaluated in experimental models of SCI for their efficacy in promoting neuroplasticity, axon regeneration, remyelination, and re-establishment of spinal circuitry to improve motor recovery.

Stem cell transplantation is a promising strategy for neural repair, either directly, by replacing the lost cells, either indirectly, by secreting neurotrophic factors / signaling molecules, which will modify cell microenvironment, making it more permissive for cellular regeneration (2). Cell-therapies alone may have a limited clinical efficacy, due to adversive environment of the host which decrease transplanted cells survival. Also, at injury site there is a lack of a physical matrix, where cells can adhere.

Biomaterials may provide structural support and/or serve as a delivery vehicle for factors to arrest growth inhibition and promote axonal growth, and are expected to stabilize the lesion site. They can mimic the natural stem cell niche's microenvironment, supporting cell growth. Designing materials to address the specific needs of the damaged central nervous system is crucial and possible with current technology (6).

Tissue-engineering techniques have developed a variety of scaffolds, made by biomaterials, used alone, incapsulated with cells or embedded with molecules, which are delivered to lesion site to achieve neural regeneration. Scaffolds are, by definition, temporary supporting structures for growing cells and tissues (2). Their aim is to provide structural -mechanic and active-trophic support to the damaged axons or cells, reducing also ths scar formation by bridging of the lesion site. Actual therapeutic strategies use scaffolds not only as volume-filling agents, but also as guidance channels for axonal regrowth and tools for cell transportation and delivery of bioactive molecules (as growth factors or proteins).

The complex therapeutic strategy of "joint treatment", using together cell transplantation, bioactive molecules delivery and biomaterials has the chance to give better results in promoting neurorestoration after spinal cord injury (7).

Various forms of scaffolds have been designed to be placed into the spinal cord lesion; the structure of these scaffolds can vary: they can be cylindrical or rectangular, resembling to a multichannel structure, or they are sponge-like, with scattered pores (8) Scaffolds are made by biomaterials, and they could be injected or implanted at lesion site. Injectable scaffolds are liquids that once injected forms gels at body temperature; their delivery is non-invasive, but they are difficult to manipulate. Implantable scaffolds are the majority, are delivered under surgical intervention at injury site (7).

Biomaterials used for scaffold fabrication must satisfy some essential criteria (3). First is biocompatibility - the material does not trigger an immune response from the host; the material should be biodegradable, degradation products should not be toxic. The biomaterial must be able to remain in place for a long time, allowing restoration. The devices should be easy to introduce in the injured spinal cord, without causing new damages (3), (8). Finally, the biomaterial should have specific mechanical and physicochemical properties - like porosity and permeability. It is suggested that the 
biomaterials used in a scaffold should have similar mechanical properties as the spinal cord.

Hydrogels are water saturated polymers, and appear as an excellent option due their physical properties which mimic the soft tissue structure and due to the possibility to adapt their chemical composition in order to integrate different molecules or proteins (3). They are extremely flexible, so they are able to be injected directly into the lesion site, where they absorbe water and expand, filling even irregularly shaped lesional cavities (8), (9). Locally, they are able to form a tridimensional structure that mimic the extracellular matrix (ECM).

The hydrogels used for scaffold fabrication can be natural or synthetic polymers, depending on the origin of the molecules being used in their fabrication.

Natural-based hydrogels are: alginate, agarose, collagen, laminin, hyaluronic acid, fibronectin, fibrin, chitosan, gelan-gum, methylcellulose, etc. These substances normally appear in natural ECM, are highly biocompatible, containing substances which are recognized by the host - signals for cell adhesion, for example, and exhibit similar properties like the soft tissues they are replacing (3), (8). The major disadvantage is that they are difficult to be sterilized, containing contaminating molecules which can induce immune responses. Also they have a fast biodegradation rate and a low mechanical resistance.

Synthetic hydrogels have a controlled synthesis process, and predictable final components. Their physical and chemical properties can be controlled, and their structure modulated in order to optimize their function. Some of the most common synthetics used in SCI repair are developed from polylactic acid (PLA)/polyglycolic acid (PGA)/ poly lacticglycolic acid (PLGA), from methacrylate (pHEMA) derivates or from polyethylene glycol (PEG) derivates.

Hydrogels are a promising therapeutical strategy in spinal cord repair. Their properties permit cell-attachment and in the same time delivery of variable agents, like growth factors or other bioactive molecules. The hydrogel is seeded with cells, and are optimized for long term survival of transplanted cells. But there is no a specific natural or synthetic polymer or combination of polymers that are perfect, research work is still ongoing in this domain, trying to determine the optimal choice for biomaterial

In biomaterials field, there is a tendancy to use nano-scale fibers instead of micro-scale fibers to achieve repair of the damaged spinal cord. The nano-fibers provide a threedimensional network, which mimic closely the native extracellular matrix, thus offering a better support for cell attachment and proliferation than traditional hydrogel structure. The axonal growth have been shown to be dependent on nano-fibres density and spatial orientation (alignment). Development of nanofibers requires specific techniques: template synthesis, phase separation, self-assembly, drawing and electrospinning. Electrospun nanofibers act as guidance channels for transplanted cells and for bioactive molecules (2). "Self-assembly peptides" are amphiphilic peptide sequences which aggregate when added to aqueous solutions and form nanofiber networks (3).

Nano-particles can also be used to administer growth factors, neurotrophic factors and antagonists of inhibitory substances, like chondroitinase $\mathrm{ABC}$ or NOGO antagonists, but they have to be injected directly in the spinal cord (8).

In the next future, researchers are trying to produce tri-dimensional scaffolds.

Despite their beneficial qualities, biomaterials alone can offer a structural support, but are not able to replace lost cells in SCI. On the other hand, cell therapies alone cannot create the complex architecture mandatory for axonal regrowth (10). Taking advantage on synergistic effects of both therapies the actual tendance is to use a combinatorial approach (11), which could offer to the injured spinal cord both the structural support, the cell source and the friendly microenvironment for functional restoration. Biomaterials acts like cell-carriers for the injury site, but also as reservoirs for growth factors or biomolecules.

Despite the new research in the field of pathogenetic mechanisms of spinal cord injury and of treatment, another factor remains under 
debate - the time: how long to continue with cell-transplantation combined with biomaterial implantation and bioactive molecules delivery?

Spinal cord injuries are complex and difficult to repair; the recent proposed therapies using biomaterials together with a combinatorial approach will improve neuroregenerating process. These are the most promising repair strategies, which will probably offer a benefit in terms of functional recovery and a lot of hope in spinal-injured patients.

\section{BIBLIOGRAPHY}

1. National Spinal Cord Injury Statistical Center, Facts and Figures at a Glance. Birmingham, AL: University of Alabama at Birmingham, 2015.

2. Tsintou M, Dalamagkas K, Seifalian AM. Advances in regenerative therapies for spinal cord injury: a biomaterials approach. Neural Regen Res. 2015 May; 10 (5): 726-742

3. Assuncao-Silva RC, Gomes ED, Sousa N, Silva NA, Salgado AJ. Hydrogels and cell based therapies in spinal cord injury regeneration. Stem Cells International, Volume 2015, ID 948040, 24 pages

4. James W. Rowland, Gregory W.J. Hawryluk, Brian Kwon, Michael G. Fehlings. Current Status of Acute Spinal Cord Injury Pathophysiology and Emerging Therapies: Promise on the Horizon.

Neurosurg Focus. 2008;25(5):E2

5. Hulsebosch CE. Recent advances in pathophysiology and treatment of spinal cord injury. Adv Physiol Educ. 2002 Dec;26(1-4):238-5

6. Haggerty AE, Oudega M. Biomaterials for spinal cord repair. Neurosci Bull. 2013 Aug;29(4):445-59.

7. Gao Y, Yang Z, Li X. Regeneration strategies after the adult mammalian central nervous system injury biomaterials. Regenerative Biomaterials,2016,115-122.

8. Siebert JR, Eade AM, Osterhout D. Biomaterial approaches to enhancing neurorestoration after spinal cord injury: strategies for overcoming inherent biological obstacles. BioMed Research International, Voluma 2015, article ID 752572, 20 pages

9. Tukmachev D, Forostyak S, Koci Z, Zavisova K, Vackova I, Vyborny $\mathrm{K}$ at al. Injectable extracellular matrix hydrogels as scaffolds for spinal cord injury repair. Tissue Eng part A. 2016 Feb;22(304): 306-17.

10. Kabu S, Gao Y, Kwon BK, Labhasetwar V. Drug delivery, cellbased therapies, and tissue engineering approaches for spinal cord injury. J Control Release. 2015 Dec 10; 219:14154.

11. Morales II, Toscano-Tejeida D, Ibarra A. Non pharmacological strategies to promote spinal cord regeneration: a view on some individual or combined approaches. Curr Pharm Des. 2016;22(6):720-7 